\title{
Radiological Outcome of Short Segment Posterior Instrumentation and Fusion for Thoracolumbar Burst Fractures
}

\author{
Truc Tam Vu ${ }^{1}$, Yuichiro Morishita ${ }^{2}$, Itaru Yugue ${ }^{2}$, Tetsuo Hayashi ${ }^{2}$, Takeshi Maeda $^{2}$, Keiichiro Shiba $^{2}$ \\ ${ }^{1}$ Department of Spinal Pathology, Hospital for Traumatology-Orthopedics, Ho Chi Minh, Vietnam \\ ${ }^{2}$ Department of Orthopedic Surgery, Spinal Injuries Center, Iizuka, Japan
}

Study Design: Retrospective study.

Purpose: To evaluate the radiological outcome of the surgical treatment of thoracolumbar burst fractures by using short segment posterior instrumentation (SSPI) and fusion.

Overview of Literature: The optimal surgical treatment of thoracolumbar burst fractures remains a matter of debate. SSPI is one of a number of possible choices, yet some studies have revealed high rates of poor radiological outcome for this SSPI.

Methods: Patients treated using the short segment instrumentation and fusion technique at the Spinal Injuries Center (lizuka, Fukuoka, Japan) from January 1, 2006 to July 31, 2012 were selected for this study. Radiographic parameters such as local sagittal angle, regional sagittal angle, disc angle, anterior or posterior height of the vertebral body at admission, postoperation and final observation were collected for radiological outcome evaluation.

Results: There were 31 patients who met the inclusion criteria with a mean follow-up duration of 22.7 months (range, 12-48 months). The mean age of this group was 47.9 years (range, 15-77 years). The mean local sagittal angles at the time of admission, post-operation and final observation were $13.1^{\circ}, 7.8^{\circ}$ and $14.8^{\circ}$, respectively. There were $71 \%$ good cases and $29 \%$ poor cases based on our criteria for the radiological outcome evaluation. The correction loss has a strong correlation with the load sharing classification score (Spearman rho $=0.64, p<0.001$ ).

Conclusions: The loss of kyphotic correction following the surgical treatment of thoracolumbar burst fracture by short segment instrumentation is common and has a close correlation with the degree of comminution of the vertebral body. Patients with high load sharing scores are more susceptible to correction loss and postoperative kyphotic deformity than those with low scores.

Keywords: Short segment instrumentation; Thoracolumbar burst fractures; Correction loss; Degree of comminution; Load sharing classification

\section{Introduction}

Thoracolumbar burst fracture is one of the most common traumatic injuries of the entire human spine and as such, there is always some debate around the indications of operative treatment $[1,2]$ as well as the optimal method of surgery for this type of fracture. The anterior approach and instrumentation, once considered the gold standard

Received Oct 25, 2014; Revised Oct 31, 2014; Accepted Nov 16, 2014

Corresponding author: Truc Tam Vu

Department of Spinal Pathology, Hospital for Traumatology-Orthopedics,

929 Tran Hung Dao Street, 5th District, Ho Chi Minh, Vietnam

Tel: +84-839237007, Fax:+84-839236554, E-mail: tamtruc240384@yahoo.com 
for restoring the weight bearing capacity of the fractured vertebra, has several drawbacks including being time consuming, a difficult technique, demanding as well as posing potential risks of major vascular damage $[3,4]$. With remarkable improvements in instrument quality, the posterior approach alone can obviate the need for reconstruction of the anterior and middle columns of the fractured vertebra. The short segment posterior instrumentation and fusion is the current trend as it can reduce the blood loss, preserving segmental motion with an acceptable anatomic and functional outcome [5-13]. However, there are also reports of high percentages of instrumentation failure $[14,15]$.

We performed short segment posterior instrumentation and fusion treatment on fractured vertebra with reinforcement by pedicle screw augmentation for almost every case of thoracolumbar burst fracture. The purpose of this retrospective study is to evaluate and analyze the radiological outcome of this technique.

\section{Materials and Methods}

\section{Study population}

From January 2006 to July 2012, a total of 31 fresh thoracolumbar (T11 to L2) burst fracture patients (21 males and 10 females) with an average age of 48.0 years (range, 15-77 years) were treated surgically. All patients underwent plain X-rays, computed tomography (CT), magnetic resonance imaging (MRI) and neurologic examination by spine surgeons at the time of admission. All patients were treated with short segment posterior instrumentation and fusion and were monitored for at least 12 months. All patients had neurological complications following surgery.

All patients were evaluated using comprehensive AO classification for thoracolumbar fracture [16] and load sharing classification [17].

This study was approved by our institution review board and informed consent was obtained from all patients.

\section{Surgical methodology}

We performed emergency operations on patients within a couple of hours following admission. Patients were positioned prone on the Jackson frame in order to reduce the intra-abdominal pressure and to create a positional reduction effect on the fracture site. Conventional longitudinal incision at the midline was routinely used to expose one level above and below the fractured vertebra. Care was taken to protect the surrounding soft tissue from exposure to and damage by the pedicle screw. Posterior fusion was performed with local bone harvested by the spinous process without laminectomy decompression even when the stenotic degree of the spinal canal was more than 50\% of its normal value. Reduction of the retropulsed fragment was performed indirectly by distraction of connecting rods.

The rehabilitation program was started the day after surgery. External fixation using orthosis was prescribed for patients during 3 months postoperatively.

\section{Radiological evaluation}

The radiological parameters include local sagittal angle (LSA), regional sagittal angle (RSA), disc angle (DAdesignated DAa if above the fractured vertebra and DAb if, below the fractured vertebra), anterior edge height of vertebra (AH-designated AHa if above the fractured vertebra, AHfx for, the fractured vertebra and AHb if, below the fractured vertebra), posterior edge height of vertebra ( $\mathrm{PH}-$ designated $\mathrm{PHa}$ if above the fractured vertebra, $\mathrm{PHfx}$ for, the fractured vertebra and $\mathrm{PHb}$, if below the fractured vertebra) (Fig. 1). We defined DA as (DAa+DAb)/2, \% $\mathrm{AH}$ as $\{\mathrm{AHfx} /[(\mathrm{AHa}+\mathrm{AHb}) / 2]\}$, and $\% \mathrm{PH}$ as $\{\mathrm{PHfx} /$ $[(\mathrm{PHa}+\mathrm{PHb}) / 2]\}$.

These parameters were taken at the time of admission, postoperation and final observation. At the final observation, we took functional plain X-rays (flexion and extension lateral views) for screening the dynamic instability of the fracture site-indirect sign of non-union and implant failure. We evaluated the instability of the fracture site based on the criteria of poor outcome widely accepted for radiological outcome evaluations which include a correction loss of more than $10^{\circ}$ of the LSA value at the final observation compared with postoperation, pedicle screw(s) or rod(s) breakage, any sign of pedicle screw loosening (persistence of radiolucency outline of the screw at the final observation) or pulled-out screw and more than a $5^{\circ}$ change in LSA with flexion and extension views $(\triangle \mathrm{LSA})$ at the final observation.

\section{Statistical analysis}

Data were analyzed using the program STATA ver. 12 

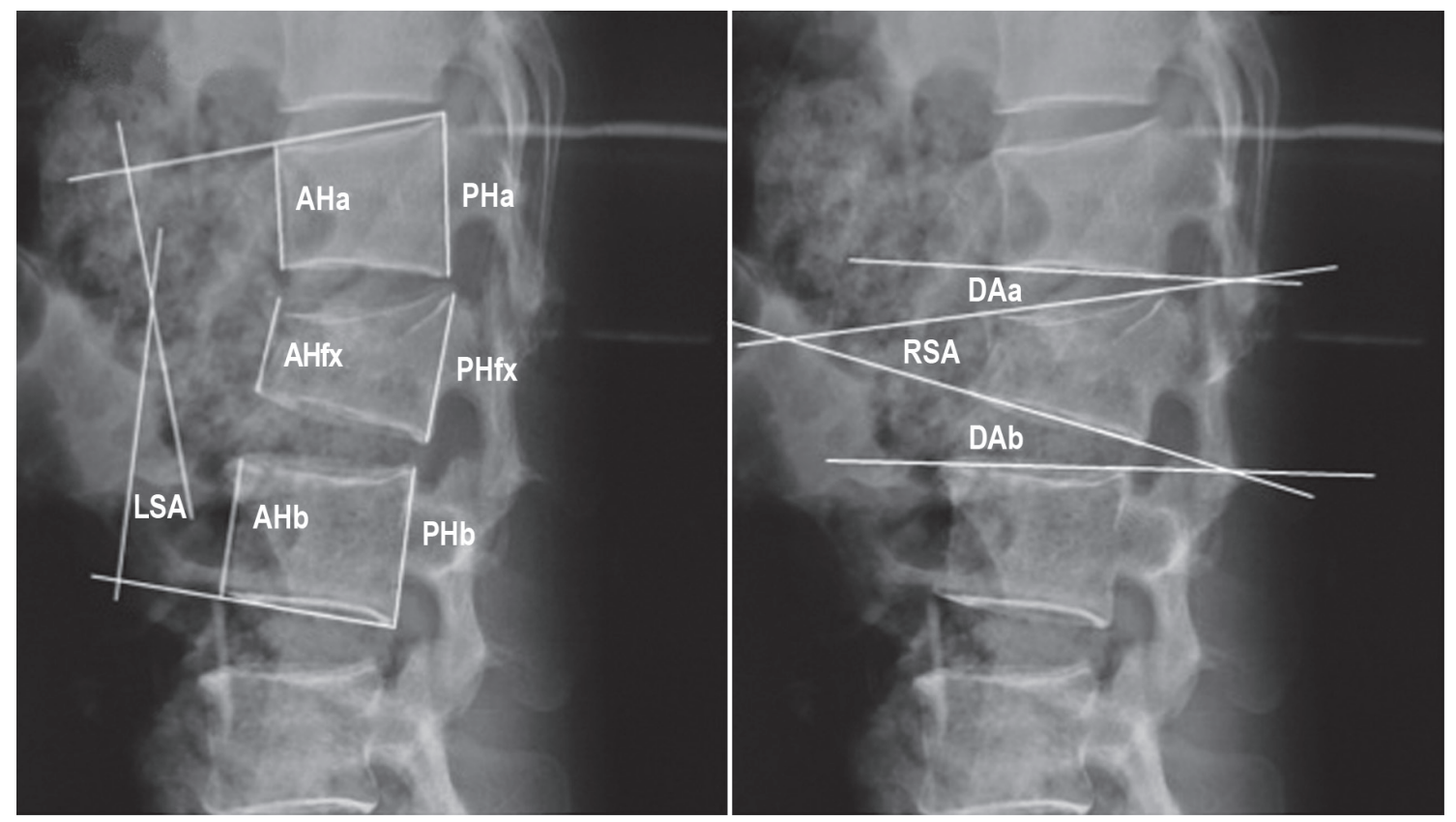

Fig. 1. Parameters on plain X-rays: local sagittal angle (LSA), regional sagittal angle (RSA), disc angle (DA-designated DAa if above the fractured vertebra and DAb if, below the fractured vertebra), anterior edge height of vertebra (AH-designated AHa if above the fractured vertebra, AHfx for, the fractured vertebra and $\mathrm{AHb}$ if, below the fractured vertebra), posterior edge height of vertebra (PH-designated $\mathrm{PHa}$ if above the fractured vertebra, $\mathrm{PHfx}$ for, the fractured vertebra and $\mathrm{PHb}$, if below the fractured vertebra).

Table 1. Radiological parameters evaluated at the admission, postoperation, and final observation

\begin{tabular}{lccc} 
Radiological parameters & Admission & Postoperation & Final observation \\
\hline Local sagittal angle $\left(^{\circ}\right)$ & 13.1 & 7.8 & 14.8 \\
\hline Regional sagittal angle $\left(^{\circ}\right)$ & 15 & 10.1 & 12.4 \\
\hline Disc angle $\left(^{\circ}\right)$ & 13.1 & 12.3 & 8.5 \\
\hline $\mathrm{AH}(\%)$ & 70.9 & 85.2 & 77.7 \\
\hline $\mathrm{PH}(\%)$ & 93.7 & 95.9 & 95.1 \\
\hline
\end{tabular}

$\mathrm{AH}$, anterior edge height of fractured vertebra; $\mathrm{PH}$, posterior edge height of fractured vertebra.

(StataCorp LP, College Station, TX, USA). Mann-Whitney $U$-test, Student $t$-test, Kruskall-Wallis test and Spearman's rank correlation test were used for statistical analysis. A p-value of less than 0.05 was considered statistically significant.

\section{Results}

\section{Demographic}

The mean follow-up duration was 22.7 months (range, 12-48 months). The male:female ratio was approximately 2:1. The most affected vertebra was L1 (19 cases), followed by T12 (12 cases). There were 12 cases of A 3.1, 9 cases of A 3.2 and 9 of A 3.3 based on the comprehensive classification of thoracolumbar fracture [16]. The mean load sharing classification score [17] was 5.3 (range, 3-8).

\section{Radiological evaluation}

The mean LSA, RSA, DA, \%AH, and \% $\mathrm{PH}$ are presented in Table 1. All of the parameters, except DA, were significantly improved after surgery but all of the parameters except $\% \mathrm{PH}$ were significantly deteriorated at the final observation when compared with postoperation.

$\triangle$ LSA showed no significant correlation with either sex or age ( $p=0.23,0.59$, respectively). Also, no significant correlations were observed between $\triangle \mathrm{LSA}$ and the level 
Table 2. Radiological outcomes of short segment posterior instrumentation and fusion for 31 patients

\begin{tabular}{|c|c|c|c|}
\hline \multicolumn{2}{|c|}{ Poor outcome } & No. $(\%)$ & Total $(\%)$ \\
\hline \multicolumn{2}{|l|}{-} & $22(71)$ & 71 \\
\hline \multirow[t]{4}{*}{+} & LSA $>10^{\circ}$ & $8(26)$ & 29 \\
\hline & Screw/rod breakage & $1(3)$ & \\
\hline & Screw loosening & 0 & \\
\hline & $\Delta \mathrm{LSA}$ (flexion-extension) $>5^{\circ}$ & 0 & \\
\hline
\end{tabular}

LSA, local sagittal angle.

Table 3. The correlation between the radiological outcome and the load sharing classification score

\begin{tabular}{lccr} 
Load sharing score & Failure & Success & Total \\
$\geq 7$ & 6 & 3 & 9 \\
$<7$ & 3 & 19 & 22 \\
Total & 9 & 22 & 31 \\
\hline
\end{tabular}

Odds ratio $=(6 \times 19) /(3 \times 3)=12.67$.

$p=0.007$ (Fischer exact test).

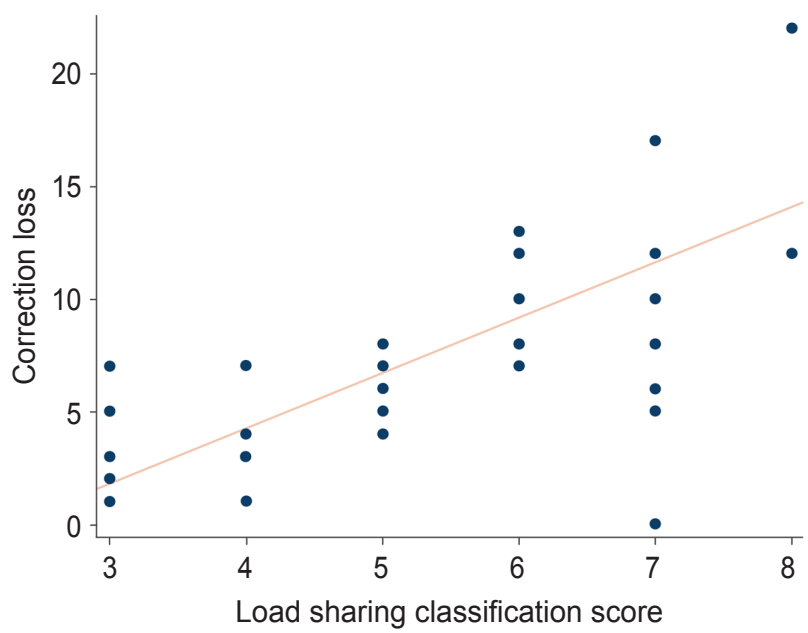

Fig. 2. The correlation between load sharing classification score and correction loss (Spearman rho $=0.64 ; p<0.001$ ).

of injury or the AO classification ( $p=0.87$ and 0.81 , respectively). However, a strong correlation was observed between $\triangle$ LSA and the load sharing classification score ( $p=0.0001$; Spearman's rho=0.64) (Fig. 2).

For the radiological outcome evaluations which indicate the instability of the fracture site, the radiographic results of short segment instrumentation are presented in Table 2. $71.0 \%$ of the patients satisfied the criteria for good radiographic outcomes while $29.0 \%$ failed to increase LSA by more than $10^{\circ}$ and/or had screw/rod breakage. There were no subjects who demonstrated screw loosening or dynamic instability evaluated by $\triangle$ LSA.

Table 3 shows the correlation between the radiological outcome and the load sharing classification score. A load sharing score of more than 7 points tended to demonstrate the failed radiological outcome with an odds ratio of 12.67 .

\section{Discussion}

In the early era of spinal instrumentation, posterior approach alone was proven to be inadequate for maintaining stability of the injured spine and yielded poor results in both the anatomical and functional aspects $[8,11,14,15]$. However, with the development of metallurgic technology and instrumentation design, pedicle screws nowadays seem to obviate the need for anterior approach.

Long segment instrumentation is strong enough to stabilize the spine. However, the fused spine becomes less flexible and more susceptible to low back pain. Therefore, short segment posterior instrumentation is thought to be a better option able to stabilize the fractured vertebra efficiently. It is also a simple technique and allows for more segmental motion of the spine. Age, osteoporosis and degree of comminution of the vertebral body are risk factors for poor surgical outcomes $[9,10]$.

In our study, kyphotic deformity was corrected to a 
considerable extent with posterior instrumentation and indirect reduction. However, the correction of kyphotic deformity which was evaluated by LSA and RSA was gradually lost during the follow up period. Although the indirect reduction by ligamentotaxis had a positive effect on the wedging deformity of the fractured vertebra, preservation of the correction might be difficult. The large bone defect created inside the fractured vertebra after height restoration had been speculated to be the most important cause of that correction loss. Moreover, the evolution of the intervertebral disc height may contribute to the loss of kyphotic correction. During the correction maneuver, the distracting effects by ligamentotaxis influenced mainly the bone, not the discs as the disc angles did not change after reduction. At the final observation, both the disc angles and the disc heights decreased in value. Several authors reported similar observations and it is believed that this decrease correlates with the posttraumatic degeneration of the discs above and below the fractured vertebra $[18,19]$.

In our results, the correction loss and the load sharing score had a strong correlation and a load sharing classification score of more than 7 points tended to demonstrate the failed radiological outcome with an odds ratio of 12.67. These results affirm previous studies [15,20] that have shown that a load sharing classification score of more than 7 points is an important risk factor in the development of losing initial anatomical restoration.

McLain et al. [15] recorded 52.5\% of poor postoperative radiological results in their research and suggested that short segment instrumentation may be inadequate for thoracolumbar burst fracture treatment. On the other hand, Lee and Sung [13] reported only $12.5 \%$ of poor results in their study. We believe that our results satisfied an acceptable percentage (29.0\%; 9 cases out of 31 ) of poor radiographical outcomes. All of these 9 cases of poor results demonstrated instrumentation failure in the caudal pedicle screws. In 8 cases with correction loss of more than $10^{\circ}$ at the final observation, the upper screws were almost in good positions. The caudal screws, on the other hand, sank downward into the vertebral body or were partially pulled out. Other cases with broken screws were also observed at the caudal screw. There are few reports focusing on whether the cranial or caudal pedicle screws have a higher risk of instrumentation failure. Our results suggest that the caudal pedicle screws might have a higher risk of instrumentation failure than the cranial screws.
The thoracolumbar spine is the junction between the less flexible thoracic spine and the more flexible lumbar spine. In the majority of cases, the cranial pedicle screws were inserted at T12, and the caudal screws at L2. The caudal pedicle screws might receive further sharing force than the cranial screws, therefore, caudal screws might exposed to a higher risk of failure. Based on our results, the subjects with load sharing classification scores of more than 7 points should extend their internal fixation using instrumentation (pedicle screws or offset hooks) to caudal segments. Even subjects with load sharing classifications of less than 7 points should adopt longer length and wider diameter of screw for the caudal pedicle screws.

The radiological outcomes do not always correspond to the clinical outcomes. The relationship between radiological findings and clinical outcomes is still a matter of discussion. Therefore, using this investigation as a pilot study, further research involving a larger patient population and long-term follow-up may help resolve several issues left unanswered.

\section{Conclusions}

Loss of kyphotic correction after surgical treatment of thoracolumbar burst fracture by short segment instrumentation is common and has a close correlation with the degree of comminution of the vertebral body. Patients with high load sharing scores are more susceptible to correction loss and postoperative kyphotic deformity than those with low scores

\section{Conflict of Interest}

No potential conflict of interest relevant to this article was reported.

\section{References}

1. Stadhouder A, Oner FC, Wilson KW, et al. Surgeon equipoise as an inclusion criterion for the evaluation of nonoperative versus operative treatment of thoracolumbar spinal injuries. Spine J 2008;8:975-81.

2. Wood K, Buttermann G, Mehbod A, Garvey T, Jhanjee R, Sechriest V. Operative compared with nonoperative treatment of a thoracolumbar burst fracture without neurological deficit: a prospective, randomized study. J Bone Joint Surg Am 2003;85:773-81. 
3. Hamdan AD, Malek JY, Schermerhorn ML, Aulivola B, Blattman SB, Pomposelli FB Jr. Vascular injury during anterior exposure of the spine. J Vasc Surg 2008;48:650-4.

4. Fantini GA, Pawar AY. Access related complications during anterior exposure of the lumbar spine. World J Orthop 2013;4:19-23.

5. Crawford RJ, Askin GN. Fixation of thoracolumbar fractures with the Dick fixator: the influence of transpedicular bone grafting. Eur Spine J 1994;3:45-51.

6. Yung AW, Thng PL. Radiological outcome of short segment posterior stabilisation and fusion in thoracolumbar spine acute fracture. Ann Acad Med Singapore 2011;40:140-4.

7. Mahar A, Kim C, Wedemeyer M, et al. Short-segment fixation of lumbar burst fractures using pedicle fixation at the level of the fracture. Spine (Phila Pa 1976) 2007;32:1503-7.

8. Dick W, Kluger P, Magerl F, Woersdorfer O, Zach G. A new device for internal fixation of thoracolumbar and lumbar spine fractures: the 'fixateur interne'. Paraplegia 1985;23:225-32.

9. Sapkas G, Kateros K, Papadakis SA, Brilakis E, Macheras G, Katonis P. Treatment of unstable thoracolumbar burst fractures by indirect reduction and posterior stabilization: short-segment versus longsegment stabilization. Open Orthop J 2010;4:7-13.

10. Kim HS, Kim SW, Ju CI, Lee SM, Shin H. Short segment fixation for thoracolumbar burst fracture accompanying osteopenia: a comparative study. J Korean Neurosurg Soc 2013;53:26-30.

11. Roy-Camille R, Roy-Camille M, Demeulenaere C. Osteosynthesis of dorsal, lumbar, and lumbosacral spine with metallic plates screwed into vertebral pedicles and articular apophyses. Presse Med 1970;78:1447-8

12. Sargin S, Ucar BY, Necmioglu S, Bulut M, Gem M.
Clinical and radiological results of posterior instrumentation without fusion for thoracolumbar fractures. Afr J Pharm Pharmacol 2011;5:819-22.

13. Lee YS, Sung JK. Long-term follow-up results of short-segment posterior screw fixation for thoracolumbar burst fractures. J Korean Neurosurg Soc 2005;37:416-21.

14. Kramer DL, Rodgers WB, Mansfield FL. Transpedicular instrumentation and short-segment fusion of thoracolumbar fractures: a prospective study using a single instrumentation system. J Orthop Trauma 1995;9:499-506.

15. McLain RF, Sparling E, Benson DR. Early failure of short-segment pedicle instrumentation for thoracolumbar fractures: a preliminary report. J Bone Joint Surg Am 1993;75:162-7.

16. Magerl F, Aebi M, Gertzbein SD, Harms J, Nazarian S. A comprehensive classification of thoracic and lumbar injuries. Eur Spine J 1994;3:184-201.

17. McCormack T, Karaikovic E, Gaines RW. The load sharing classification of spine fractures. Spine (Phila Pa 1976) 1994;19:1741-4.

18. Kerttula LI, Serlo WS, Tervonen OA, Paakko EL, Vanharanta HV. Post-traumatic findings of the spine after earlier vertebral fracture in young patients: clinical and MRI study. Spine (Phila Pa 1976) 2000;25:1104-8.

19. Tschoeke SK, Hellmuth M, Hostmann A, et al. Apoptosis of human intervertebral discs after trauma compares to degenerated discs involving both receptormediated and mitochondrial-dependent pathways. J Orthop Res 2008;26:999-1006.

20. Dai LY, Jiang LS, Jiang SD. Conservative treatment of thoracolumbar burst fractures: a long-term follow-up results with special reference to the load sharing classification. Spine (Phila Pa 1976) 2008;33:2536-44. 